\title{
High dose methylprednisolone in severe acute transverse myelopathy
}

Guillaume Sébire, Henri Hollenberg, Laurence Meyer, Gilbert Huault, Pierre Landrieu, Marc Tardieu

\begin{abstract}
No effective treatment has been shown for patients with acute transverse myelopathy. In an open study five children with severe acute transverse myelopathy were treated with intravenous methylprednisolone and compared with a historical group of 10 patients. The results show that in the methylprednisolone treatment group compared with the historical group of 10 patients: the median time to walk independently was significantly reduced (23 $v 97$ days); the proportion of patients with a full recovery within 12 months was significantly higher ( $80 v 10 \%)$; all patients had complete motor recovery within one year in contrast with only two of 10 patients in the historical group; and serious adverse effects did not occur. This pilot study suggests that high dose methylprednisolone is effective in the treatment of acute transverse myelopathy.

(Arch Dis Child 1997;76:167-168)
\end{abstract}

Keywords: acute transverse myelopathy; myelitis; paraplegia; methylprednisolone.

Acute transverse myelopathy (ATM) is a severe disorder lasting several months and often leading to a poor outcome. ${ }^{1-4}$ The annual incidence in the Israeli population has been estimated as $1.34 / 10^{6}{ }^{4}$ The presumed immune mediated physiopathological mechanism for this disease, the high risk of residual neurological deficiency, and the lack of effective treatment prompted us to evaluate the effect of intravenous methylprednisolone (MPIV) in five children and to compare the outcome with that of a historical control group of 10 consecutive patients from the same centre. ${ }^{1-8}$ Because of the risk of side effects we chose to treat patients with a severe form of the disease characterised by complete flaccid paralysis of the legs; this has been associated with a high risk of poor outcome. ${ }^{3}$ All the children (age range 2-14 years) fulfilled the classical criteria for the diagnosis of $\mathrm{ATM}^{134}$ : briefly, no antecedent neurological illness, acute onset of bilateral spinal cord dysfunction (clinical features summarised in table 1), and exclusion of patients with trauma, irradiation, or compressive spinal cord lesion (magnetic resonance imaging in eight patients, myelography in two patients, Queckenstedt test in two patients, and no investigation in three patients owing to concomitant acute optic neuritis).

\section{Patients and methods}

Five children consecutively admitted were treated with MPIV (Solumedrol) at a dosage of $1 \mathrm{~g} / 1.73 \mathrm{~m}^{2}$ (except one patient who received $0.5 \mathrm{~g} / 1.73 \mathrm{~m}^{2} /$ day) every day for three (two patients) or five consecutive days (three patients), followed in four of the five patients by prednisone by mouth (Cortancyl, $1 \mathrm{mg} / \mathrm{kg} /$ day) for a total treatment duration of 14 days (MPIV group). Only children whose parents gave informed consent took part in the study. Their outcome was compared with that of a historical control group of 10 consecutive patients from the same centre receiving either no specific treatment (four patients) or low doses of corticosteroids which, according to other studies, ${ }^{13}$ do not affect the outcome (four patients treated with adrenocorticotropic hormone, two patients treated with prednisone, and one patient treated with both.

The main outcome criteria were the median time to walk independently and the proportion of patients with a full recovery within three or 12 months (recovery occurred mostly within 12 months in previously published papers). ${ }^{1-4}$ Percentages were compared using Fisher's exact test. The median time to walk independently in each group was compared using the Kruskal-Wallis test. Student's $t$ test was used to compare the mean ages and the durations of follow up in each group.

\section{Results}

The clinical and paraclinical characteristics of the two groups, especially those suggested to be of prognostic value, namely back pain and the duration and severity of the initial course, are described in table $1 .{ }^{13}$ With the exception of sex ratio and follow up duration, these characteristics did not statistically differ between the two groups. The mean delay between the beginning of the disease and MPIV administration was nine days (range 5-16 days). In the MPIV group the median time to recovery of independent walking was $23 v 96.5$ days in the control group $(p=0.01)$. The percentage of patients recovering independent walking within four weeks was $100 \%$ in the MPIV group compared with $20 \%$ in the control group $(p=0.007)$. The percentage of patients with a full recovery within three months was $60 \%$ in the MPIV group and $0 \%$ in the control group $(p=0.02)$, whereas the proportion of patients with a full recovery within 12 months was $80 \%$ in the MPIV group and $10 \%$ in the control group $(\mathrm{p}=0.01)$.

The one patient in the MPIV group with a disability at 12 months had minor sphincter 
Table 1 Characteristics of the two groups of patients

\begin{tabular}{lll}
\hline Clinical and paraclinical features & $\begin{array}{l}\text { MPIV group } \\
(n=5)\end{array}$ & $\begin{array}{l}\text { Control group } \\
(n=10)\end{array}$ \\
\hline Year of observation (range) & $1994-5$ & $1975-93$ \\
Mean age (years) & 9.2 & 8.6 \\
Male/female ratio & $5 / 0$ & $3 / 7$ \\
Clinical features & $2 / 5$ & $4 / 10$ \\
Back pain & 3.1 & 5 \\
Mean delay before maximum deficit (days) & $0 / 5$ & $2 / 10$ \\
Hyperacute onset (maximum deficit within 12 hours) & D9-D12 & C4-D12 \\
Sensory level ${ }^{\star}$ & $5 / 5$ & $10 / 10$ \\
Complete flaccid paraplegia & $4 / 5$ & $10 / 10$ \\
$\quad$ Sphincter distubances & $2 / 5$ & $2 / 10$ \\
Supramedullar symptoms $\dagger$ & $4 / 5$ & $7 / 10$ \\
No with increased cerebrospinal fluid cell count & $4 / 5$ & $1 / 10$ \\
Full recovery within one year & 1.2 & 4.1 \\
Mean follow up (years) $\ddagger$ & & \\
\hline
\end{tabular}

* Sensory level located between C4 and D9 in $80 \%$ of the patients from the control group. † Optic neuritis (two patients in the control group, one patient in the MPIV group), brainstem or hemispheric white matter lesions (one patient in each group).

$\ddagger$ The follow up was at least 12 months except in two patients (six and 10 months) from the treated group who had a rapid and full recovery.

disturbance (precipitant micturition). In contrast, in the control group, patients who had not recovered at 12 months had severe (incontinence or retention of urine in seven patients associated with paraplegia in two wheelchair dependent patients) or moderate disabilities (mild bladder sphincter disturbances associated with independent but deficient gait in two patients). The proportion of patients with complications, namely urinary tract infection or bedsores, was $20 \%$ in the MPIV group and $40 \%$ in the control group $(p=0.10)$. No patient had a recurrence of neurological symptoms.

\section{Discussion}

This pilot study suggests that treatment with MPIV may be highly effective in children with severe forms of ATM.

The aetiology of ATM in childhood remains unsettled, but a postinfectious autoimmune process is strongly suspected owing to the winter clustering, the association with a previous infection, the increased cerebrospinal fluid cell count, and some anatomical studies showing infiltration of spinal cord white matter by immune cells. ${ }^{1-7}$ The myelin sheath seems to be the target of the pathological process, as suspected clinically by the location of occasional supramedullar symptoms, such as optic neuritis, and confirmed by anatomical studies showing areas of demyelination in the spinal cord. ${ }^{3568}$ Peripheral blood lymphocytes from patients with ATM showed a significant proliferative response when cultured in vitro in the presence of myelin proteins such as myelin basic protein. ${ }^{7}$ Thus ATM might be, at least in part, the consequence of a cell mediated autoimmune response towards a component of the myelin sheath. The likely efficacy of MPIV treatment for optic neuritis and multiple sclerosis led us to study this treatment in the severe forms of ATM in children. ${ }^{9}{ }^{10}$ The mechanism of action of MPIV is still unknown. In peripheral blood MPIV induced a transient lymphopenia with a significant reduction in CD4 positive cells. ${ }^{11}{ }^{12}$ Interleukin-6 regulates the production of antibodies and the cytotoxic activity of $T$ cells. Interestingly, in vitro, glucocorticoids downregulated the production of interleukin- 6 by brain and spinal cord macrophages (microglial cells). ${ }^{13}$

The results of this study suggest that MPIV treatment is effective in shortening the length of the disease and in improving outcome at one year in severe forms of ATM. The baseline characteristics of patients did not differ between the two groups except for sex ratio and level of spinal cord lesion, which probably do not affect outcome. ${ }^{3}$ Despite selection of the severe forms, the proportion of patients with sequelae at two years in our control group $(80 \%)$ was only slightly higher than that from other reported large paediatric series (62$66 \%$ ), reinforcing the value of this historical control group. ${ }^{2}$

A final conclusion, however, can only come from a randomised double blind study of MPIV $v$ placebo which, owing to the large differences observed in this pilot study, might require relatively few patients. The potentially adverse effects of high dose MPIV should also be assessed against the burden of sequelae in untreated ATM.

1 Dunne H, Hopkin IJ, Shield LK. Acute transverse myelopathy in childhood. Dev Med Child Neurol 1986;28:198-204. Paine RS, Byers RK. Transverse myelopathy in childhood. Am f Dis Child 1953;85:151-63.

3 Ropper AH, Poskanzer DC. The prognosis of acute and Ropper AH, Poskanzer DC. The prognosis of acute and
subacute transverse myelopathy based on early signs and subacute transverse myelopathy based

4 Berman M, Feldman S, Alter M, Zilber N, Kahana E. Acute transverse myelitis: incidence and etiologic considerations. Neurology 1981;31:966-71.

5 Greenfield JO, Turner JWA. Acute and subacute necrotic myelitis. Brain 1939;62:227-52.

6 Hoffman HL. Acute necrotic myelopathy. Brain 1955;78: 377-91.

7 Abramsky O, Teitelbaum D. The autoimmune features of acute transverse myelopathy. Ann Neurol 1977;2:36-40.

8 Scully RE, Mark EJ, McNeely WF, McNeely BU. Case records of the Massachusetts General Hospital. N Engl f Med 1995;333:1485-93.

9 Beck RW, Cleary PA, Anderson MM, et al. A randomized, controlled trial of corticosteroids in the treatment of acute optic neuritis. N Engl F Med 1992;326:581-8.

10 Goodin DS. The use of immunosuppressive agents in the treatment of multiple sclerosis: a critical review. Neurology 1991;41:980-5.

11 Miller JJ. Prolonged use of large intravenous pulses in the rheumatic diseases of children. Pediatrics 1980;65:989-94.

12 Silverman ED, Myones BL, Miller JJ. Lymphocyte subpopulation alterations induced by intravenous megadose pulse methyl-prednisolone. F Rheumatol 1984;11:287-90.

13 Sébire G, Delfraisssy JF, Demotes-Mainard J, et al. Interleukin-13 and interleukin-4 act as interleukin-6 inducers in human microglial cells. Cytokine 1996;8:636-41. 